\title{
Simulations of nanograting-assisted light coupling in GaN planar waveguide
}

\author{
R. Dylewicz - R. A. Hogg - R. Airey - R. Paszkiewicz • \\ P. Bientsman · S. Patela
}

Received: 23 September 2010 / Accepted: 14 July 2011 / Published online: 30 July 2011

(C) The Author(s) 2011. This article is published with open access at Springerlink.com

\begin{abstract}
The numerical simulations of nanogratings integrated with gallium nitride $(\mathrm{GaN})$ planar waveguides as well as the experimental in-coupling results are presented. A simulation tool based on the eigenmode expansion method and advanced boundary conditions provided a rigorous model of 400-nm-period grating couplers. A full-vectorial Maxwell solver allowed performing a number of simulations with varying grating parameters, where coupling efficiency, reflection and transmission characteristics of device were calculated. Gratings with different etch depths and arbitrary shapes were simulated using a staircase approximation, with an optimized number of steps per single slope. For the first time, an impact of dry etch processing on GaN coupler efficiency was evaluated, due to the inclusion of the sloped sidewalls, with regard to the technological constrains. Finally, the experimental results in the visible spectrum region $(\lambda=633 \mathrm{~nm})$, for 400 -nm-deep gratings etched in GaN waveguide,
\end{abstract}

Electronic supplementary material The online version of this article (doi:10.1007/s11082-011-9485-4) contains supplementary material, which is available to authorized users.

R. Dylewicz $\cdot$ R. Paszkiewicz $\cdot$ S. Patela

Faculty of Microsystem Electronics and Photonics, Wroclaw University of Technology, Wroclaw, Poland

R. Dylewicz

Optoelectronics Research Group, Department of Electronics and Electrical Engineering, University of Glasgow, Glasgow, UK

R. A. Hogg · R. Airey

Department of Electronic and Electrical Engineering, EPSRC National Center for III-V Technologies, University of Sheffield, Sheffield, UK

P. Bientsman

Department of Information Technology, Ghent University/IMEC, Ghent, Belgium

Present Address:

R. Dylewicz $(\varangle)$

Lam Research AG, Villach, Austria

e-mail: rafal.dylewicz@lamresearch.com 
were presented together with theoretical data for binary and trapezoidal profiles of a grating, for different optical mode profiles $\left(\mathrm{TE}_{0} \div \mathrm{TE}_{3}\right.$ modes $)$.

Keywords Integrated optics · Planar waveguide · Grating coupler · Gallium nitride · Numerical simulations · Dry etching process - Semiconductor processing · III-V semiconductor materials

$\begin{array}{ll}\text { Abbreviations } \\ \text { GaN } & \text { Gallium nitride } \\ \text { EEM } & \text { Eigenmode expansion method } \\ \text { PICs } & \text { Photonic integrated circuits } \\ \text { 2-D } & \text { Two-dimensional } \\ \text { LEDs } & \text { Light emitting diodes } \\ \text { CAMFR } & \text { CAvity Modelling FRamework } \\ \text { FDTD } & \text { Finite difference time-domain } \\ \text { PMLs } & \text { Perfectly matched layers } \\ \text { GPL } & \text { General public license } \\ \text { SOI } & \text { Silicon-on-insulator } \\ \text { LT } & \text { Low-temperature } \\ \text { MOCVD } & \text { Metaloorganic chemical vapour deposition } \\ \text { PECVD } & \text { Plasma-enhanced chemical vapor deposited } \\ \text { ff } & \text { Filling factor } \\ \text { SEM } & \text { Scanning electron microscope } \\ \text { ICP } & \text { Inductively coupled plasma } \\ \text { EBL } & \text { Electron beam lithography }\end{array}$

\section{Introduction}

III-V-Nitride compounds ( $\mathrm{GaN}, \mathrm{InN}$ and $\mathrm{AlN})$ are interesting and prospective materials for future optoelectronics applications. These new materials can be indispensable in violet and UV spectral regions, but they are also very useful for generation and detection of blue and green light. All III-Nitrides have a direct energy band-gap, a valuable feature which decreases their optical attenuation by at least one order of magnitude as compared to indirect bandgap semiconductors, like e.g. SiC (Pankove 1999). For the same reason, the efficiency of photon emission and absorption is also higher. There is also a possibility of forming solid solutions with InN and AlN, which allows tailoring optical and electrical properties. GaN exhibits a high mechanical and thermal stability, which makes it very useful for high-temperature electronic and optoelectronic purposes, i.e. for ultraviolet and blue emitting GaN-based lasers.

The realization of grating-assisted coupling into/from GaN planar waveguides can be useful for the investigation of the optical properties of a material, i.e. quality assessment of the gallium nitride growth technique. Such an approach allows the study of waveguide modes and the measurement of refractive index and thickness of a layer, material loss (in a dual-coupler setup), characterization of optical anisotropy (Dylewicz et al. 2009b) and the refractive index distribution within the layer. Due to the small dimensions of the grating and its integration with the waveguiding layer, a sample of almost arbitrary shape and size can be thoroughly measured and characterized. It also gives fabrication possibilities 
for photonic integrated circuits (PICs) and provides the device compactness. On the contrary, prism coupling, where discreet optical elements are used, limits the sample size and rules out the integration scheme. The two-dimensional (2-D) approach presented here may be useful for advanced simulations and further fabrication of other low-dimensional structures in $\mathrm{GaN}$, e.g. gratings and 2-D photonic crystals. Enhanced light extraction from the GaNbased light emitting diodes (LEDs) is reported with the application of integrated sub-micron or nano-sized structures (Wierer et al. 2004; Shakya et al. 2004). Photonic crystal formation on the top surface of the LED device supports the effect of optical modes extraction from a waveguide, which is basically the same out-coupling phenomenon as presented in this paper.

Quick and accurate design optimization process can be based upon the presented calculations, as the usefulness of eigenmode expansion method (EEM) is shown in this paper. Another task was to introduce CAvity Modelling FRamework (CAMFR) software in some more details, so that interested readers will be benefited if they can reproduce the results presented here. Last but not least, a correlation between fabrication feasibility, to transfer low-dimensional features to GaN, and coupler efficiency was presented. To the best of our knowledge, this is the first time when a strong dependence between nanograting efficiency and dry etch quality, mainly due to the inclusion of sloped sidewalls, was numerically proved.

\section{Simulation model}

The fabrication process of submicron-sized periodic structures requires a large number of subsequent technological stages to obtain a ready-to-use passive device. Fabrication is timeconsuming, expensive and involves an advanced semiconductor facility. A possible approach to reduce time and effort is an accurate computer simulation of a desired structure. One of the commonly used methods for a simulation of the periodic structures of a finite extent is a finite difference time-domain (FDTD) method (Cryan et al. 2005; Dridi and Bjarklev 1999). The FDTD method can be used to tackle a large class of the problems and precisely solves the equations governing the device behavior, but at the cost of computational resources. Grating couplers of the finite dimensions were also investigated using a coupled-mode theory (Hardy et al. 1989; Hoekstra 2000). The main constraint related to the coupled mode theory is that it will model only gratings of a modest refractive index contrast. In order to obtain reliable information about a waveguide-based grating coupler performance with a minimum expenditure of computing memory and running time CAvity Modelling FRamework (CAMFR) software was used. This simulation tool is a full-vectorial Maxwell solver based on an eigenmode expansion (EEM) and advanced boundary conditions including the Perfectly Matched Layers (PMLs). The usability of the EEM has been proved mainly in the field of photonics to simulate the behavior of wavelength-scale photonic structures like photonic crystal devices (Bogaerts et al. 2002; Bienstman et al. 2001), VCSEL lasers (Bienstman and Baets 2001), resonant-cavity light-emitting diodes (Bienstman and Baets 2000) and grating couplers (Taillaert et al. 2002, 2004). EEM leads to a much more compact representation of the field, which drastically improves the simulation accuracy and computing speed. CAMFR is freely available software from http://camfr.sourceforge.net distributed under an open source license, i.e. General Public License (GPL) scheme. It is worth to mention that although grating couplers were initially calculated with use of CAMFR software (Taillaert et al. 2002, 2004), those analyses were related to the structures fabricated in entirely different material base, i.e. in silicon-on-insulator (SOI) waveguides. This implies different refractive index contrast than for GaN/sapphire wafer, waveguide layer thickness, dry etch possibilities, etc. so that those references are not very relevant to GaN-based structures. 
(a)

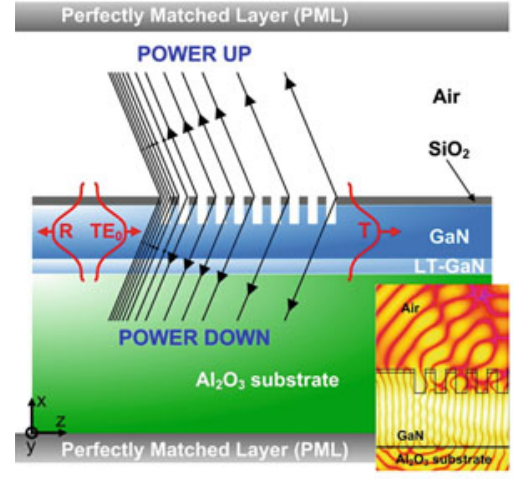

(b)

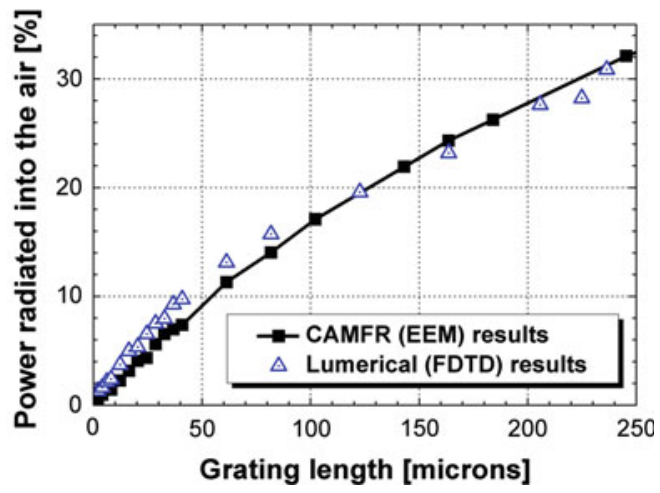

Fig. 1 GaN planar waveguide with integrated 400-nm-period nanogratings: a schematic representation of the performed 2-D simulations, inset: Ey electric field distribution for $\mathrm{TE}_{0}$ mode (log scale), multimedia file available; b comparison of 2-D results achieved with the EEM method (CAMFR software) and a commercial FDTD package (Lumerical)

The simulation scheme of a waveguide-based GaN coupler is presented in Fig. 1a. The guided wave (e.g. $\mathrm{TE}_{0}$ optical mode), propagating from the left to the right, is coupled out of the waveguide. The modal energy is radiated out both in the air (Power up) and downwards in the substrate direction (Power down), as shown in the inset in Fig. 1 and additionally in the animation of electric field distribution (Online Resource 1), for the fundamental $\mathrm{TE}_{0}$ optical mode, when a periodic corrugation in $\mathrm{GaN}$ waveguide appears. Here, reflectivity $R$ represents the energy transfer and the coupling between electromagnetic modes that are propagating in opposite directions. $T$ is the transmission of the energy through the grating structure along the $+z$-direction. The density of the flux lines in Fig. 1a indicates the theoretical intensity variation in the output beams and therefore the resulting beam profile is expected to be nearly exponential.

Both theoretical and experimental $\mathrm{GaN}$ planar waveguides consist of a $\mathrm{SiO}_{2}$ upper cladding $(60 \mathrm{~nm})$, a $1.47-\mu \mathrm{m}$-thick GaN waveguide core and a low-temperature (LT) GaN buffer $(\sim 20 \mathrm{~nm})$ on sapphire $\left(\mathrm{Al}_{2} \mathrm{O}_{3}\right)$ substrate. GaN samples were grown by metaloorganic chemical vapour deposition (MOCVD) at Wroclaw University of Technology, Poland. The refractive index of the plasma-enhanced chemical vapor deposited (PECVD) $\mathrm{SiO}_{2}$ layer was measured to be $n=1.482$ for a laser wavelength $\lambda=632.8 \mathrm{~nm}$. The refractive index of gallium nitride layer grown by MOCVD was experimentally (Dylewicz et al. 2009b) found to be $n_{G a N}=2.349$. The presence of an additional LT-GaN layer was included in model. The buffer layer, which is grown during MOCVD process prior to the main layer, is characterized by a lower refractive index than the following GaN film (Dogheche et al. 1999). The change of optical properties at the GaN-sapphire interface is related to the fact that during the early stage of growth, many defects are introduced into a film, which can lead to islanding and columnar growth. Generally, the LT-GaN buffer layer has been found to have a low value of $n(2.10<n<2.25)$ in the wavelength range $400<\lambda<850 \mathrm{~nm}$ (Bergmann et al. 1999). Therefore, the value of $n \sim 2.175$ was taken here for the calculation purposes. Birefringence of sapphire substrate, with an ordinary refractive index for TE-polarized light $n=1.7661$ was taken into consideration. The thickness of both $\mathrm{Al}_{2} \mathrm{O}_{3}$ substrate and air surroundings, which is the distance between perfectly matched layers (PML) and a waveguide, were evaluated from the optimization of simulation parameters. In this experiment only multi-mode 
GaN waveguides, supporting up to eight TE-polarized modes, were investigated. This is due to a common problem with MOCVD growth of thin GaN layers $(<1 \mu \mathrm{m})$ of high optical and structural quality, e.g. with a low defect density, low surface roughness, etc. Grating couplers are most commonly used for single mode waveguides, e.g. 220-nm-thick silicon-on-insulator (SOI) planar waveguides (Taillaert et al. 2002, 2004). Thin waveguide structure means in practice high coupling efficiency as strong overlap between optical field and a grating structure is evidenced for fundamental mode, almost regardless of an etch depth. On the other hand, multimode waveguides are beneficial for well-controlled excitement of optical modes to study guided wave propagation parameters and thus optical properties of MOCVD gallium nitride (Dylewicz et al. 2009b). The most reliable results are obtained in the multimode structures, while for a waveguide structure supporting only one optical mode the measurements have to be performed for at least two different wavelengths. For that purpose even a low efficiency coupling behaviour in a multimode structure proved to be sufficient to derive GaN optical properties, i.e. $60^{\circ}$ periodic in-plane anisotropy of refractive indices.

In the EEM-based calculations 1- $D$ gratings for TE-polarized light at $\lambda=632.8 \mathrm{~nm}$, in wavelength regions near the band edge, were considered. The 1st order gratings with the constant period of $\Lambda=400 \mathrm{~nm}$ and filling factor $f f$ (duty cycle) of $50 \%$, as for the fabricated devices, were used in all performed calculations. Grating period was set to fulfill the coupling condition for TE/TM polarized light, for a laser wavelength of $\lambda=632.8 \mathrm{~nm}$, although grating periodicity was not optimized for a maximum coupling efficiency.

In order to check the correctness of CAMFR results before providing more advanced simulations, a comparison between two different methods and software packages was performed. Simulation results achieved with freely available EEM method (CAMFR tool) and commercial FDTD package (FDTD Solutions by Lumerical, Canada) are presented in Fig. $1 \mathrm{~b}$. Grating coupler efficiency results for a simple binary grating are in a good agreement for a wide range of simulated device length, varying from 2 to $250 \mu \mathrm{m}$.

\section{Simulation results for grating coupler etch depth}

Several different factors, i.e. grating etch depth, should be taken into consideration when designing an appropriate coupler device. The dependence of the etch depth on coupler behavior for an ideal (binary) grating and different TE optical modes was investigated for a

(a)

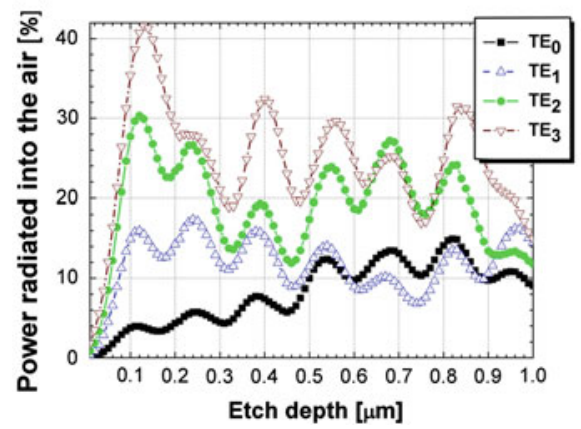

(b)

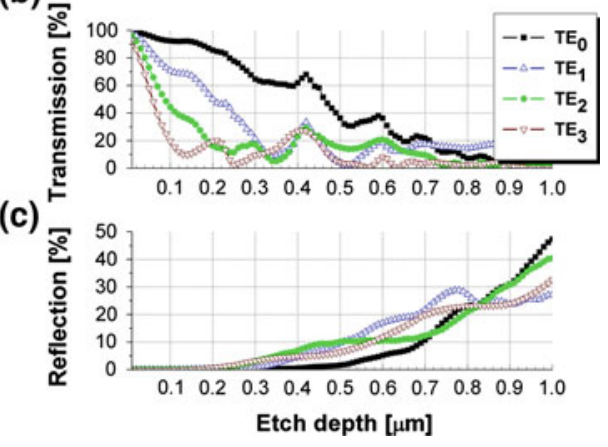

Fig. 2 Simulation of a binary gratings with height (etch depth) varied, for different TE modes: a power radiated in the air, $\mathbf{b}$ transmission, $\mathbf{c}$ reflection of the optical power 
40- $\mu \mathrm{m}$-long device (Fig. 2). For the fundamental $\mathrm{TE}_{0}$ mode the efficiency characteristic is readily predictable, as presented in Fig. 2a. The out-coupling efficiency of the $\mathrm{TE}_{0}$ mode is increasing as a deeper grating is etched, until it reaches the maximum value for the grating height of $H=0.82 \mu \mathrm{m}$. The efficiency curves plotted for the higher order modes are not so obvious. The characteristics for $\mathrm{TE}_{2}$ (solid green line) and $\mathrm{TE}_{3}$ (dashed-dotted brown line) modes are maximal for relatively shallow gratings, i.e. etch depth $H=0.13 \mu \mathrm{m}$ for $\mathrm{TE}_{3}$ mode. The transmission of higher order modes (Fig. 2b) is rapidly decreasing for deeper structures, particularly for the grating height $H>0.3 \mu \mathrm{m}$. This effect indicates, in conjunction with reflection behavior shown in Fig. 2c, that a lot of energy is radiated downwards. The higher order TE modes are more efficiently transferred into radiated modes when a periodic perturbation is present. One possible explanation for the increased coupler efficiency is the strong field overlap between the optical field and the grating structure. The effect of mode number on a grating behavior, presented in this work, is in good agreement with the results described in (Tamir and Peng 1977; Fradin et al. 1976). Moreover, a similar behavior was noticed for an experimentally observed grating sample. The reflection of all investigated TE modes, presented in Fig. $2 \mathrm{c}$, is kept at a reasonable level $(R \leq 10 \%)$ only for a shallow and medium depth grating with $H \leq 0.5 \mu \mathrm{m}$. For deeply etched structures both reflection and coupling to the substrate may be dominant effects. Redundant reflections can be suppressed by changing the grating filling ratio, i.e. fabrication of narrower air-slot while keeping constant period $\Lambda$ can reduce the parasitic effect. Finally, an etch depth of $H=0.4 \mu \mathrm{m}$ was chosen for the fabrication of experimental devices, which is trade-off between moderate coupling efficiency for all the optical modes, low reflectivity and fabrication feasibility.

\section{Simulation results for arbitrary slope angle and shape of grating}

Grating shape and sidewall slope angle are strongly determining the performance of a coupler device. The influence of the grating profile on the coupling efficiency, transmission and reflection level of the fundamental $\mathrm{TE}_{0}$ mode was investigated for a grating length of $20 \mu \mathrm{m}$. The step approximation was applied to construct the advanced grating profiles with an optimized number of steps $S=12$ per each sloped sidewall, as shown in Fig. 3a. Grating couplers with different groove forms were investigated: lamellar (binary) gratings,

(a)

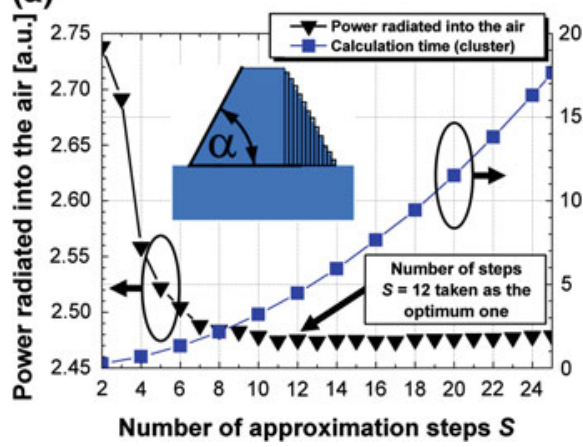

(b)

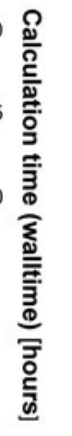

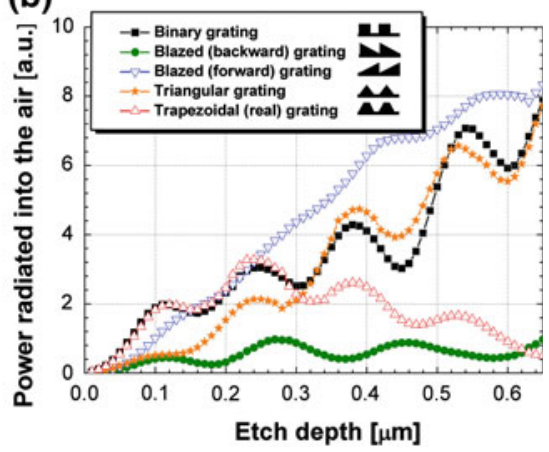

Fig. 3 Simulations of gratings with an arbitrary shape: a coupler efficiency and calculation time in a function of number of steps used in staircase approximation; $\mathbf{b}$ optical power radiated in the air for different grating coupler profiles and etch depths in GaN planar waveguide 
blazed backward and blazed forward structures, triangular-groove gratings and trapezoidal gratings, which profile is identical with that of fabricated GaN nano-devices. The sidewalls slope angle for a trapezoidal grating $\left(\alpha \sim 80^{\circ}\right)$ was assumed after analysis of scanning electron microscope (SEM) micrographs. The profile of a grating plays a significant role in the final performance of a grating coupler device (Fig. 3b). The power radiated upwards is presented in a function of a grating tooth height, for several different shapes of a groove/ridge. As theoretically expected, the most outstanding device behavior is observed for a forward blazed grating coupler. The properties of such unsymmetrical gratings differ from the performance of the symmetrical ones, e.g. lamellar gratings. An incident light is divided here into unequal efficiencies of the symmetrical diffraction orders, i.e. the efficiency in an $m=-1$ order seriously varies from that in an $m=+1$ order. A strong directional discrimination of the output radiation exists for the blazed profiles and moreover the efficiency of the forward blazed grating is diametrically opposed to the performance of the backward blazed grating. A directional discrimination is inherent in the characteristics of asymmetric profile grating couplers (Chang and Tamir 1980; Peng and Tamir 1974) and has been proved experimentally (Aoyagi et al. 1976). From a practical point of view, the comparison between the efficiency of an ideal binary grating and its realistic realization, which is trapezoidal grating, is of a particular importance. It can be noticed in Fig. 3b that for relatively shallow structures both profiles have a common behavior. The influence of the sloped sidewalls with the inclination angle $\alpha=80^{\circ}$ is not noticeable for the tooth height up to $H=0.29 \mu \mathrm{m}$. Above that value the curves are separating from each other, hence a strong divergence between results for a perfect grating and a real grating is observed.

As mentioned before, a similarity of the performance curves for shallow gratings with different profiles can be observed in Fig. 3b. The parallel behavior is noticed for the most of gratings regardless of their shape, for the etch depths up to $H \sim 0.25 \mu \mathrm{m}$. For a relatively shallow gratings the reflection, transmission (not shown here) and, to a lesser degree, the coupling efficiency is unrelated to the grating profile. The remarks done here are typical for gratings supporting only two diffraction orders, the $m=0$ and the $m=-1$ diffraction order. For gratings with only two supported orders and shallow depths this similarity is expressed in a so-called equivalence rule, which is not a rigorous theorem but an empirical rule, with a strong argument in a perturbation theory for shallow structures. The only limitation of the rule is that a centre of symmetry is present in a structure, so the profile function $f(z)$ can be expanded in Fourier sine series:

$$
f(z)=\sum_{n=0}^{n=\infty} f_{n} \sin \left(n \frac{2 \pi}{\Lambda} z\right)
$$

where $f_{n}$ is the $n$th Fourier harmonic of the profile and $\Lambda$ is a grating period. For a variety of groove profiles, when a filling factor $f f=0.5$ and a relatively low modulation depth are assumed, the equivalence rule can be safely used.

The impact of quality of submicron-sized features after fabrication with the use of dry etching technique, namely inductively coupled plasma (ICP) process, on GaN coupler efficiency is presented in Fig. 4. Nanograting efficiency is strongly related to the pattering conditions, mainly due to the inclusion of sloped sidewalls in GaN. Relative optical power coupled in the air is shown against sidewalls inclination angle $\alpha$ (as shown in inset of Fig. 3a), for five different etch depths varying from 150 to $550 \mathrm{~nm}$. Two contrary cases are shown in SEM pictures (Fig. 4b, c) and are additionally marked as "b" and "c" in Fig. 4a, where ICP dry etch conditions determined the slope angle of GaN nanogratings (Dylewicz et al. 2009a). For the 150- and 200-nm-deep couplers the maximum performance of a device is observed 

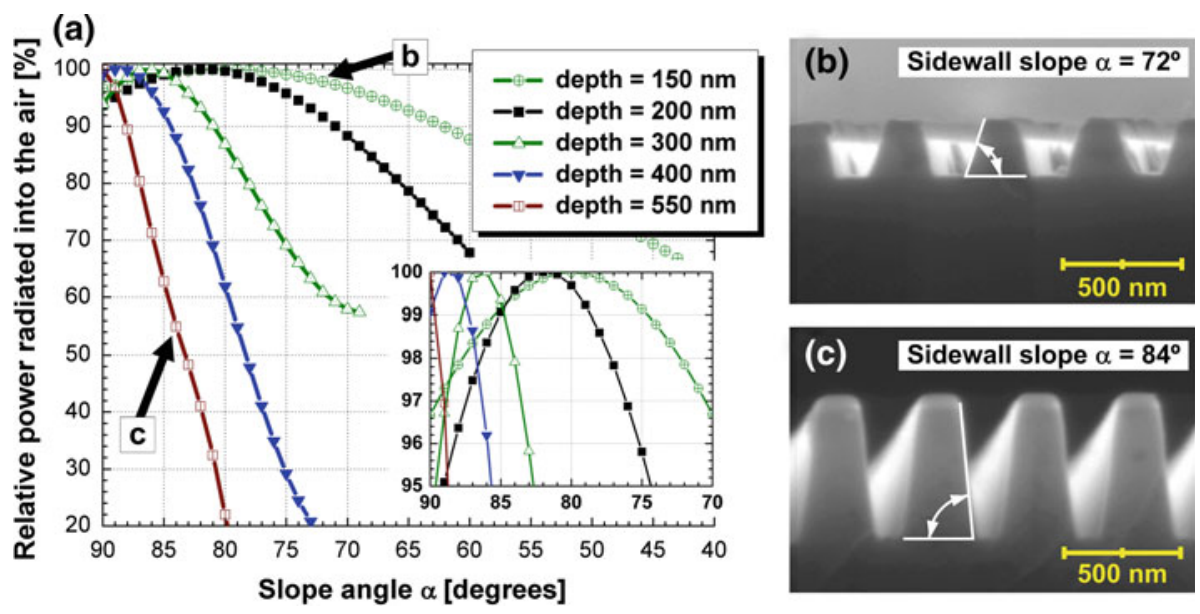

Fig. 4 Fabrication-related constrains for GaN nanogratings: a simulation results of coupler efficiency versus sidewalls slope angle $\alpha$; b SEM picture of shallow ( 150-nm-deep) grating with the slope angle of $72^{\circ}$; c SEM picture of deep ( $\sim 550$-nm-deep) grating with the slope angle of $84^{\circ}$

not for the case of perfectly vertical sidewalls $\left(\alpha=90^{\circ}\right)$, but for the sloped ones $\left(\alpha \sim 82^{\circ}\right)$. Additionally, the influence of the non-vertical sidewalls on the power radiated out is not crucial for a wide angular range of $\alpha$. As seen in Fig. 4a, the dependence of the slope angle on the coupler efficiency is much more decisive for an increased etch depth in the GaN layer. For the 400- and 550-nm-deep grating couplers the angle of the maximum performance has been shifted towards the vertical sidewalls instance. The resulting coupling behavior is here strongly dependent on the inclination angle $\alpha$ and a serious deterioration of the efficiency characteristic is therefore expected for the steep slopes.

\section{Experimental in-coupling results}

Experimental results of the in-coupling efficiency are presented in Fig. 5, together with theoretical out-coupling data calculated with the use of EEM simulations. The fabrication of real devices included optimization of both electron beam lithography (EBL) and ICP dry etching process, carried with use of $\mathrm{SiCl}_{4} / \mathrm{Cl}_{2} / \mathrm{Ar}$ gas mixture (Dylewicz et al. 2007, 2009a,b). The need for optimization of high resolution etching in GaN with use of ICP technique is due to fine structure of designed grating coupler, i.e. corrugation periodicity of $400 \mathrm{~nm}$ with ridge/groove width of approximately $200 \mathrm{~nm}$ each. The etch depth of fabricated gratings was set to $\sim 400 \mathrm{~nm}$, in order to provide sufficient coupling efficiency and minimize the perturbation of the guided modes. The ready-to-use gratings (Fig. 5a) were written over the area of $500 \mu \mathrm{m} \times 500 \mu \mathrm{m}$ and tested in an optical measurement setup with a classical diffraction mounting and TE polarized laser beam at the fixed wavelength of $\lambda=632.8 \mathrm{~nm}(\mathrm{He}-\mathrm{Ne}$ laser). Input-coupling efficiency depends on the incident beam spot size. Laser beam diameter of $\varnothing=66 \mu \mathrm{m}$ was used in the experiment, after grating efficiency was evaluated for a range of variable laser spots. Low impact of incidence conditions upon the optical measurements was therefore provided with collimated beam setup. During the measurements the laser beam was incident at the grating coupler structure at an incident angle $\Theta_{i}$, near the grating edge. By varying $\Theta_{i}$ the phase-matching conditions can be fulfilled and hence one of the diffracted 

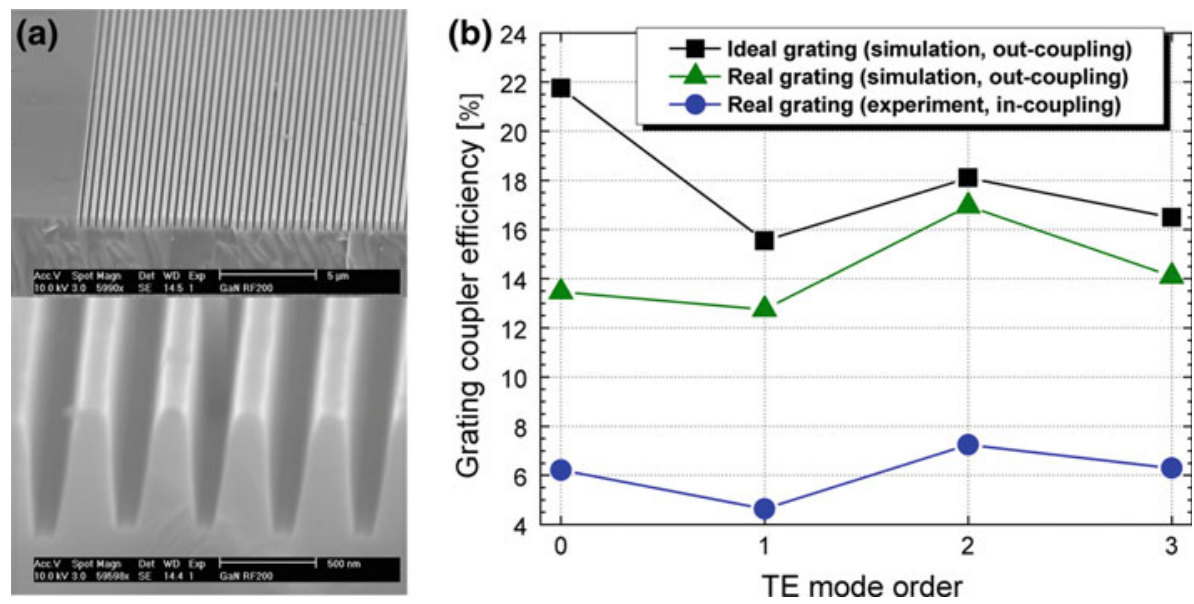

Fig. 5 Grating coupler efficiency: a SEM cross-section of the 400-nm-period grating coupler etched in a $1.47 \mu \mathrm{m}$-thick gallium nitride layer, b experimental data for in-coupling efficiency of a 400-nm-deep grating coupler compared with theoretical out-coupling values calculated for binary and trapezoidal gratings

waves will propagate in the waveguide as an optical mode excited in the structure. The estimation of input coupler efficiency is based on an energy balance criterion. The dip method, which was used in the experiment, relies on monitoring the intensity of all the beams which emerge in the setup, with the exception of a guided wave (Dakss et al. 1970; Kogelnik and Sosnowski 1970). The decrease in the intensity of these beams, observable as a dip in a trace, as $\Theta_{i}$ is varied through the resonance condition indicates that the portion of energy is being carried off by the TE optical mode, excited in a planar waveguide. Experimental results of the in-coupling efficiency for a grating coupler fabricated in a GaN waveguide are presented in Fig. 5b, where observed efficiency is noted in a function of the TE mode order. The results obtained from modeled structures are shown in two distinctive cases: an ideal binary grating coupler and a trapezoidal one with a slope angle of $\alpha=80^{\circ}$, both for $500-\mu \mathrm{m}$-long devices.

It can be seen in Fig. $5 b$ that the efficiency trends for different modes are consistent, both for the measured and the computed data, while the maximum coupling efficiency is evidenced for $\mathrm{TE}_{2}$ optical mode. As predicted from the previous simulations (Fig. 4a), the sloped sidewalls of a device resulted in a lowered coupling efficiency (comparison between theoretical out-coupling results for binary and trapezoidal grating). The diminished coupler efficiency is observed regardless of the optical mode order. The discrepancy in the amount of power radiated out, between the experimental in-coupling and theoretical out-coupling results, can be noticed.

A number of possible reasons are given to explain the difference between theory and experiment. One is the mismatch between the Gaussian input beam and the exponential profile of the grating coupler. It is related to the reciprocal theorem and the differences between in- and out-coupling. Generally, an input coupler is characterized by a lower efficiency than an output coupler due to the effect of a beam form (Tamir 1975). Reciprocity guarantees that $100 \%$ of the output efficiency will be preserved also for an input coupler if equal transverse beam energy distribution is secured in both cases. Hence, a similar response is expected only if the incident beam from $\mathrm{He}-\mathrm{Ne}$ laser has an exponential profile. Additional reasons can be related to the fabrication process, e.g. grating non-uniformity due to the stitching errors or moderate light scattering at the rough $\mathrm{GaN}$ surfaces. Sidewalls slope different from an 
expected value of $\alpha=80^{\circ}$ can also strongly affect the coupling behavior. The rapid decrease of efficiency as the slope angle falls down is presented in Fig. 4a, where a significant change of coupler performance (drop by $-53 \%$ ) is noticed when the inclination angle changes from 80 to $75^{\circ}$, for the etch depth of $400 \mathrm{~nm}$.

\section{Conclusions}

The usefulness of EEM for modeling of GaN-based nanogratings was presented in this paper, for the visible wavelength region close to the emission wavelengths of GaN-based active devices. Response of passive photonic structures, i.e. GaN waveguide-based gratings used for coupling of radiated modes, was calculated. The simulations of the out-coupling efficiency and device behavior were performed for different TE optical modes and etch depths, where effect of a shape of the grating teeth was studied. A correlation between fabrication possibilities, i.e. pattering of dense features in $\mathrm{GaN}$, and coupler efficiency was presented. It has been proven that the nanograting efficiency is strongly related to the dry etching quality, mainly due to the inclusion of sloped sidewalls in the pattering process. Finally, experimental in-coupling results were shown together with theoretical out-coupling data for binary and trapezoidal gratings, in case of a $500-\mu \mathrm{m}$-long device.

Acknowledgements This work was supported by Polish State Committee for Scientific Research during 2004-2007 under Grant 3 T11B 039 27. This work was supported in part by British Council Young Scientists Programme and EU Leonardo da Vinci Programme Future Engineers in Europe. CAMFR numerical calculations were performed at Wroclaw Centre for Networking and Supercomputing (WCSS), Wroclaw, Poland.

Open Access This article is distributed under the terms of the Creative Commons Attribution Noncommercial License which permits any noncommercial use, distribution, and reproduction in any medium, provided the original author(s) and source are credited.

\section{References}

Aoyagi, T., Aoyogi, Y., Namba, S.: High-efficiency blazed grating couplers. Appl. Phys. Lett. 29, 303304 (1976)

Bergmann, M.J., Özgür, Ü., Casey, H.C. Jr., Everitt, H.O., Muth, J.F: Ordinary and extraordinary refractive indices for $\mathrm{Al}_{x} \mathrm{Ga}_{1-x} \mathrm{~N}$ epitaxial layers. Appl. Phys. Lett. 75, 67-69 (1999)

Bienstman, P., Baets, R.: The RC ${ }^{2}$ LED: a novel resonant-cavity LED design using a symmetric resonant cavity in the out-coupling mirror. IEEE J. Quantum Electron. 36, 669-673 (2000)

Bienstman, P., Baets, R.: Optical modelling of photonic crystals and VCSELs using eigenmode expansion and perfectly matched layers. Opt. Quantum Electron. 33, 327-341 (2001)

Bienstman, P. et al.: Comparison of optical VCSEL models on the simulation of oxide-confined devices. IEEE J. Quantum Electron. 37, 1618-1631 (2001)

Bogaerts, W., Bienstman, P., Taillaert, D., Baets, R., De Zutter, D.: Out-of-plane scattering in photonic crystal slabs. IEEE Photon. Tech. Lett. 13, 565-567 (2002)

Chang, K.C., Tamir, T.: Simplified approach to surface-wave scattering by blazed dielectric gratings. Appl. Opt. 19, 282-288 (1980)

Cryan, M.J., Wong, D.C.L., Craddock, I.J., Yu, S., Rorison, J., Railton, C.J.: Calculation of losses in 2D photonic crystal membrane waveguides using the 3D FDTD method. IEEE Photon. Tech. Lett. 17, 58-60 (2005)

Dakss, M.L., Kuhn, L., Heidrich, P.F., Scott, B.A.: Grating couplers for efficient excitation of optical guided waves in thin films. Appl. Phys. Lett. 16, 523-525 (1970)

Dogheche, E., Belgacem, B., Remiens, D., Ruterana, P., Omnes, F.: Interface properties of $\mathrm{Al}_{x} \mathrm{Ga}_{1-x} \mathrm{~N} / \mathrm{AlN}$ heterostructures from optical waveguiding information. Appl. Phys. Lett. 75, 3324-3326 (1999) 
Dridi, K.H., Bjarklev, A.: Optical electromagnetic vector-field modeling for the accurate analysis of finite diffractive structures of high complexity. Appl. Opt. 38, 1668-1678 (1999)

Dylewicz, R., Hogg, R.A., Fry, P.W., Parbrook, P.J., Airey, R., Tahraoui, A., Patela, S.: Inductively coupled plasma etching of $\mathrm{GaN}$ using $\mathrm{SiCl}_{4} / \mathrm{Cl}_{2} / \mathrm{Ar}$ for submicron-sized features fabrication. Phys. Stat. Sol. C 4, 2634-2637 (2007)

Dylewicz, R., Patela, S., Hogg, R.A., Fry, P.W., Parbrook, P.J., Airey, R., Tahraoui, A.: Low-dimensional waveguide grating fabrication in $\mathrm{GaN}$ with use of $\mathrm{SiCl}_{4} / \mathrm{Cl}_{2} / \mathrm{Ar}$-based inductively coupled plasma dry etching. J. Electron. Mater. 38, 635-639 (2009a)

Dylewicz, R., Patela, S., Hogg, R.A., Fry, P.W., Parbrook, P.J., Airey, R., Tahraoui, A.: In-plane optical anisotropy of GaN refractive index in visible light region. IEEE Photon. Tech. Lett. 21, 966-968 (2009b)

Fradin, D.W., Cheo, P.K., Peng, S.T., Tamir, T.: Effects of imperfections on the efficiency of optical couplers. J. Opt. Soc. Am. 66, 292 (1976)

Hardy, A., Welch, D., Streifer, W.: Analysis of second order gratings. IEEE J. Quantum Electron. 25, 20962105 (1989)

Hoekstra, H.: Coupled mode theory for resonant excitation of waveguiding structures. Opt. Quantum Electron. 32, 735-758 (2000)

Kogelnik, H., Sosnowski, T.P.: Holographic thin film couplers. Bell Syst. Tech. J. 49, 1602-1609 (1970)

Pankove, J.I.: GaN: from fundamentals to applications. Mater. Sci. Eng. B61-62, 305-309 (1999)

Peng, S.T., Tamir, T.: Directional blazing of waves guided by asymmetrical dielectric gratings. Opt. Commun. 11, 405-409 (1974)

Shakya, J., Kim, K.H., Lin, J.Y., Jiang, H.X.: Enhanced extraction in III-Nitride ultraviolet photonic crystal light-emitting diodes. Appl. Phys. Lett. 85, 142-144 (2004)

Taillaert, D., Bogaerts, W., Bienstman, P., Krauss, T.F., Van Daele, P., Moerman, I., Verstuyft, S., De Mesel, K., Baets, R.: An out-of-plane grating coupler for efficient butt-coupling between compact planar waveguides and single-mode fibers. IEEE J. Quantum Electron. 38, 949-955 (2002)

Taillaert, D., Bienstman, P., Baets, R.: Compact efficient broadband grating coupler for silicon-on-insulator waveguides. Opt. Lett. 29, 2749-2751 (2004)

Tamir, T: Beam and waveguide couplers. In: Tamir, T. (ed.) Integrated Optics, Springer, Berlin (1975)

Tamir, T., Peng, S.T.: Analysis and design of grating couplers. Appl. Phys. 14, 235-254 (1977)

Wierer, J.J., Krames, M.R., Epler, J.E., Gardner, N.F., Craford, M.G., Wendt, J.R., Simmons, J.A., Sigalas, M.M.: InGaN/GaN quantum-well heterostructure light-emitting diodes employing photonic crystal structures. Appl. Phys. Lett. 84, 3885-3887 (2004) 